\title{
Sequential Use of Testosterone Gel and Growth Hormone in Expected Poor Responders and those with Previous Poor Assisted Reproductive Technology Outcomes: A Pilot Study
}

\author{
Sathya Balasubramanyam
}

\begin{abstract}
Introduction: Poor responders have suboptimal outcomes following conventional in vitro fertilization/intracytoplasmic sperm injection treatment. There is some evidence that transdermal testosterone and growth hormone may help in improving live birth rates in this group.
\end{abstract}

Aim: To present a case series of women who had sequential transdermal testosterone and growth hormone treatment in view of their being expected poor responders or with a history of previous poor oocyte or embryo quality.

Setting: Private assisted reproduction clinic.

Materials and methods: A total of 24 women underwent 30 cycles of controlled ovarian stimulation. Ten patients out of 24 had previous poor assisted reproductive technology outcomes, of which 4 were poor responders. Fourteen were expected poor responders. The women used approximately $1.2 \mathrm{gm}$ of transdermal testosterone from day 5 to 25 along with a standard oral contraceptive pill. Growth hormone was given at 8 units/day subcutaneously from day 2 along with the gonadotropins in the antagonist protocol.

Results: The mean age of the women was 34.92 years $( \pm 3.6)$. The average duration of subfertility was $7.54( \pm 4.005)$ years. The mean antral follicle count was $9( \pm 3.28)$ and the mean anti-Mullerian hormone level was $1.2 \mathrm{ng} / \mathrm{mL}( \pm 0.56)$. The mean number of eggs collected was $8( \pm 5.45)$. Number of mature (M2) eggs was $6.6( \pm 4.5)$ Mean number of eggs fertilized was 5.04 ( \pm 4.03$)$; clinical pregnancy rate was $8 / 24(33.3 \%)$ and ongoing pregnancy rate was $4 / 24(16.6 \%)$.

Conclusion: This case series shows an encouraging clinical pregnancy rate. The reduced ongoing pregnancy rate probably reflects the suboptimal gamete quality. Further randomized controlled trials (RCTs) are needed to assess the efficacy of sequential transdermal testosterone and growth hormone therapy in poor responders.

Clinical significance: The ongoing pregnancy rate in this group with poor prognosis seems encouraging, and further well-designed RCTs would help in assessing the merits of this sequential therapy.

Clinical Director

Department of Reproductive Medicine, Cloudnine Fertility Center, Chennai Tamil Nadu, India

Corresponding Author: Sathya Balasubramanyam, Clinical Director, Department of Reproductive Medicine, Cloudnine Fertility Center, Chennai, Tamil Nadu, India, Phone: +918605009999 e-mail: sathyabala2000@gmail.com
Keywords: Growth hormone, In vitro fertilization, Pilot study, Poor responder, Transdermal testosterone.

How to cite this article: Balasubramanyam S. Sequential Use of Testosterone Gel and Growth Hormone in Expected Poor Responders and those with Previous Poor Assisted Reproductive Technology Outcomes: A Pilot Study. Int J Infertil Fetal Med 2017;8(1):1-4

Source of support: Nil

Conflict of interest: None

Date of received: 2-8-2016

Date of acceptance: 5-9-2016

Date of publication: May 2017

\section{INTRODUCTION}

The outcome of in vitro fertilization (IVF) cycles depends significantly on the number of eggs collected. ${ }^{1}$ There is some evidence to suggest that the use of testosterone gel in the preceding cycle might help in increasing the number of eggs collected. ${ }^{2}$ There is also limited evidence that the use of growth hormone increases live birth rates in poor responders undergoing IVF/intra-cytoplasmic sperm injection (ICSI) cycles. ${ }^{3}$ In our present series, we have used sequentially testosterone gel and growth hormone in expected poor responders and those with previous poor assisted reproductive technology (ART) outcomes.

\section{MATERIALS AND METHODS}

A total of 24 women including expected poor responders based on age, antral follicle count (AFC), and/serum anti-Mullerian hormone (AMH) levels and those with previous poor ART outcome (poor oocyte or embryo quality) were offered sequential testosterone gel and growth hormone. The study period was from July 2015 to June 2016 in a private assisted reproduction clinic. The women applied approximately $1.2 \mathrm{gm}$ of testosterone gel on their shoulders from day 5 to 25 along with an oral contraceptive pill for the same duration. Controlled ovarian stimulation was done with the gonadotrophinreleasing hormone antagonist protocol using recombinant 
follicle stimulating hormone (FSH) 225-300-450 units per day (Gonal F, Merc Serono) and injection cetrorelix $0.25 \mathrm{mg}$ subcutaneously (Cetrotide, Merc Serono) from day 5 of controlled ovarian stimulation until the day of human chorionic gonadotropin ( $\mathrm{hCG}$ ) trigger. The trigger for ovulation was with injection hCG 5,000 units intramuscularly (IM) and injection triptorelin $0.2 \mathrm{mg}$ subcutaneously when at least two follicles reached an average follicular size of $18 \mathrm{~mm}$. Growth hormone 8 units per day was given subcutaneously from day 2 of the cycle until the day of the hCG trigger. Number of eggs, their maturity, morphology, fertilization, cleavage, clinical pregnancy, and ongoing pregnancy were noted. Based on morphology, oocytes were classified into three groups - normal, suboptimal, or poor. The oocytes with smooth endoplasmic reticulum clusters and giant oocytes were classified as having poor quality. Those with other morphological variants like central pitting, wide perivitelline space, debris in perivitelline space, fragmented polar body were classified as suboptimal. The others were classified as normal. Clinical and ongoing pregnancy rates were calculated per patient and other data are per oocyte retrieval. Correlation graphs between number of follicles $>16 \mathrm{~mm}$ with number of metaphase 2 (M2) oocytes; number of M2 oocytes with number of good, suboptimal, and poor morphology oocytes; and number of good morphology embryos were plotted. A comparison of the mean number of eggs collected in a previous cycle without testosterone and growth hormone combination in the poor ART outcome group and the current cycle with these adjuvants was made by the Mann-Whitney U test. Pregnancy outcomes were tabulated (Table 1).

\section{RESULTS}

A total of 24 women underwent 30 cycles of controlled ovarian stimulation. Ten women out of 24 had previous poor ART outcome, of which 4 were poor responders. Fourteen were expected poor responders. The mean age of the women was 34.92 years $( \pm 3.6)$. The average duration of subfertility was $7.54( \pm 4.005)$ years. The mean AFC was $9( \pm 3.28)$ and the mean AMH level was $1.2 \mathrm{ng} / \mathrm{mL}$

Table 1: Pregnancy outcome

\begin{tabular}{ll}
\hline Subject & Outcome \\
\hline $\begin{array}{l}\text { Mrs A Electron } \\
\text { paramagnetic } \\
\text { resonance (EPR) }\end{array}$ & First trimester miscarriage \\
Mrs B (EPR) & \\
Mrs K Post partum & Delivered term singleton \\
angiopathy (PPA) & Delivered twins dichorionic diamniotic \\
Mrs C (EPR) & Teterm intrauterine growth restriction \\
Mrs S (PPA) & Ectopic pregnancy \\
Mrs P (PPA) & Missed miscarriage \\
Mrs N (EPR) & Ongoing pregnancy third trimester \\
Mrs AN (EPR) & An embryonic gestation \\
\hline
\end{tabular}

Table 2: Patient characteristics

\begin{tabular}{ll}
\hline Patient characteristics & Mean number \\
\hline Mean age (years) & $34.92( \pm 3.6)$ \\
Mean duration of subfertility (years) & $7.54( \pm 4.005)$ \\
Mean AFC & $9.0( \pm 3.28)$ \\
Mean AMH $(\mathrm{ng} / \mathrm{mL})$ & $1.2( \pm 0.56)$ \\
\hline
\end{tabular}

Table 3: Results of ovum pick up and embryo transfer

\begin{tabular}{ll}
\hline Ovum pick up and embryo transfer & Mean number \\
\hline Eggs collected & $8( \pm 5.45)$ \\
M2 oocytes & $6.6( \pm 4.5)$ \\
Eggs fertilized & $5.04( \pm 4.03)$ \\
Embryos transferred & $2.6( \pm 1.0)$ \\
\hline
\end{tabular}

$( \pm 0.56)$. The mean AFC in the expected poor responder group was $7.7( \pm 1.49)$. The mean AFC in the previous poor ART outcome group was $11( \pm 4.8)$. Table 2 summarizes the patient characteristics.

The mean number of eggs collected was $8( \pm 5.45)$. Number of mature (M2) eggs was $6.6( \pm 4.5)$. The mean number of normal morphology eggs was $5.8( \pm 4.89)$. The proportion of normal morphology oocytes was 154/222 (69.3\%). Proportion of suboptimal oocytes was 56/222 (25.2\%). Proportion of poor morphology oocytes was 12/222 (5.4\%). Mean number of eggs fertilized was $5.04( \pm 4.03)$. Mean number of embryos transferred was 2.6( \pm 1.0$)$ (Table 3). There was a fair correlation between number of follicles more than $16 \mathrm{~mm}$ on the day of hCG trigger and number of M2 oocytes. There was a good correlation between number of M2 oocytes and the number of good morphology oocytes. There was poor correlation between the number of M2 oocytes and the number of suboptimal and poor morphology oocytes. Mean number of eggs collected in the previous cycle in the poor ART outcome group was 5.1 $( \pm 3.6)$, while the mean number of eggs in the current cycle was $11.1( \pm 6.4)$. The difference was statistically significant, $\mathrm{p}=0.0375$ ( $\mathrm{U}$ value 16.5 and $\mathrm{Z}$ score -2.075 ).

Fertilization rate was $162 / 222(72.9 \%)$. The clinical pregnancy rate was $8 / 24(33.3 \%)$ and ongoing pregnancy rate is $4 / 24(16.6 \%)$.

\section{DISCUSSION}

The success rates in IVF/ICSI in poor responders are significantly less compared with normo- or hyperresponders. In recent years, studies have reported increased live birth rates following the use of growth hormone during controlled ovarian stimulation. ${ }^{4,5}$ Some studies have failed to demonstrate significant benefit. ${ }^{6,7}$ However, there is not much consensus on what the optimal dose is (4-20 units). Also, the question of when to start and how long to give also remains unclear. Starting growth hormone in the luteal phase of the preceding cycles and on day 2 or 3 along with gonadotrophins have been described. ${ }^{7}$ 
Improvement of oocyte quality is proposed by growth hormone upregulating its own receptors on the oocyte and increasing functional mitochondria. ${ }^{8}$ There are some studies that show an improvement in oocyte and embryo quality and not so much in oocyte number. ${ }^{9,10}$ There is, hence, a school of thought to start growth hormone in late follicular phase until the day preceding the oocyte retrieval. A recent randomized controlled trial (RCT) showed that growth hormone given as an adjuvant in the long protocol gave more eggs and better fertilization rates compared with other protocols, but the improvement in clinical pregnancy rates did not reach significance. ${ }^{11}$

The mean AFC was 9 as our group also included patients with normal ovarian reserve but previous poor oocyte or embryo quality. The mean AMH was lower at $1.2 \mathrm{ng} / \mathrm{mL}$, as we usually do not check AMH levels in expected normoresponders. Hence, there is disparity between the mean AFC, which is done for all patients, and AMH levels, which are done in patients with lower AFC or previous poor ovarian response. In our series, we have given 8 units of growth hormone per day from day 2 until the day of hCG trigger. This was a randomly decided dose. We also added injection triptorelin $0.2 \mathrm{mg}$ subcutaneously, so that we have an FSH surge as well, thereby reducing the number of immature oocytes. This is routine practice in our unit to reduce the probability of failure of the triggering injection to act. The proportion of M2 oocytes was $85.05 \%(222 / 261)$. The mean number of eggs was significantly higher in the testosterone-growth hormone-treated cycle compared with the previous cycle without these adjuvants. However, the gonadotrophin dosage could not be compared as the information was not available for some of the previous cycles done elsewhere.

The recent Cochrane review on the role of androgens in poor responders concluded that there may be an increase in live birth on adding testosterone gel in the preceding cycle. The quality of evidences is reported as moderate. ${ }^{2}$ An earlier meta-analysis had shown a significant increase in clinical pregnancy and live birth rates with the use of transdermal testosterone in poor responders. ${ }^{12}$ There are studies which report no benefit as well. ${ }^{13}$ Some experts question the physiological basis of transdermal testosterone for short periods. ${ }^{14}$ The mechanism of action has not been clearly elucidated. It has been postulated that increasing intrafollicular androgens would upregulate FSH receptors in the granulosa cells and increase follicular growth. ${ }^{15}$

As growth hormone and testosterone gel appear to be acting through different pathways, we thought of giving them sequentially to our patients who were expected poor responders or those with previous history of poor response(less than three eggs collected) and poor egg/ embryo quality.
We found the clinical pregnancy rate per patient to be significantly high at $33.3 \%$. The ongoing pregnancy rate came down to $16.6 \%$. This suggests that overall improvement is present, if at all transient. Growth hormone and testosterone cannot do much from the aneuploidy perspective.

Nevertheless, the ongoing pregnancy rate in this group with poor prognosis seems encouraging, and further well-designed RCTs would help in assessing the merits of this sequential therapy. Weakness in this series is the small number, inclusion of both expected poor responders, and previous poor ART outcomes. The outcome of this study merely reflects the possibility of limited benefit and the need for further investigation of this combination in the management of poor responders.

\section{CONCLUSION}

This case series shows an encouraging clinical pregnancy rate. The reduced ongoing pregnancy rate probably reflects the suboptimal gamete quality. Further RCTs are needed to assess the efficacy of sequential transdermal testosterone and growth hormone therapy in poor responders.

\section{REFERENCES}

1. Sunkara SK, Rittenberg V, Raine-Fenning N, Bhattacharya S, Zamora J, Coomarasamy A. Association between the number of eggs and live birth in IVF treatment: an analysis of 400135 treatment cycles. Hum Reprod 2011 Jul;26(7):1768-1774.

2. Nagels HE, Rishworth JR, Siristatidis CS, Kroon B. Androgens (dehydroepiandrosterone or testosterone) for women undergoing assisted reproduction. Cochrane Database Syst Rev 2015 Nov;6(11):CD009749.

3. Duffy JM, Ahmad G, Mohiyiddeen L, Nardo LG, Watson A. Growth hormone for in vitro fertilization. Cochrane Database Syst Rev 2010 Jan 20;(1):CD000099.

4. Bayoumi YA, Dakhly DM, Bassiouny YA, Hashish NM. Addition of growth hormone to the microflare stimulation protocol among women with poor ovarian response. Int J Gynaecol Obstet 2015 Dec;131(3):305-308.

5. Lattes K, Braaesco M, Gomez M, ChecaMA. Low-dose growth hormone supplementation increases clinical pregnancy rate in poor responders undergoing in vitro fertilization. Gynecol Endocrinol 2015 Jul;31(7):565-568.

6. Bassiouny YA, Dakhly Dm, Bayoumi YA, Hashish NM. Does the addition of growth hormone to the in vitro fertilization/ intra cytoplasmic sperm injection antagonist protocol improve outcomes in poor responders? A randomized, controlled trial. Fertil Steril 2016 Mar;105(3):697-702.

7. Dunne C, Seethram K, Roberts J. Growth hormone supplementation in the luteal phase before microdose GnRH antagonist flare protocol for in vitro fertilization. J Obstet Gynaecol Can 2015 Sep;37(9):810-815.

8. Weall BM, Alsamerria S, Conceicao J, Yovich JL, Almah G. A direct action for growth hormone in improving oocyte quality 
in poor responder patients. Reproduction 2015 Feb;149(2): 147-154.

9. Bosch E, Labarta E, Kolibianakis E, Rosen M, MeBldrum D. Regimen of ovarian stimulation affects oocyte and therefore embryo quality. Fertil Steril 2016 Mar;105(3):560-570.

10. de Ziegler D, Streuli I, Meldrum DR, Chapron C. The value of growth hormone supplements in ART for poor ovarian responders. Fertil Steril 2011 Nov;96(5):1069-1076.

11. Dakkly DM, Bayoumi YA, Gad Allah SH. Which is the best IVF/ICSI protocol to be used in poor responders receiving growth hormone as an adjuvant treatment? A prospective randomized trial. Gynecol Endocrinol 2016;32(2):116-119.

12. Bosdou JK, Venetis CA, Kolibianakis EM, Toulis KA, Goulis DG, Zepiridis L, Tarlatzis BC. The use of androgens or androgen-modulating agents in poor responders undergoing in vitro fertilization: a systematic review and meta-analysis. Hum Reprod Update 2012 Mar-Apr;18(2): 127-145.

13. Bosdou JK, Venetis CA, Dafopoulos K, Zepiridis L, Chatzimeletiou K, Anifandis G, Mitsoli A, Makedos A, Messinis IE, Tarlatzis BC, et al. Reproduction trans dermal testosterone pretreatment in poor responders undergoing ICSI: a randomized clinical trial. Hum Reprod 2016 May;31(5):977-985.

14. Polyzos NP, Davis SR, Drakopoulos P, Humaidan P, De Geyter C, Vega Ag, Martinez F, Evangelou E, van de Vijver A, Smitz J, et al. Testosterone for poor ovarian responders: lessons from ovarian physiology. Reprod Sci 2016 Aug;pii:1933719116660849.

15. Sunkara SK, Coomarasamy A, Arlt W, Bhattacharya S. Should androgen supplementation be used for poor ovarian response in IVF?. Hum Reprod 2012 Mar;27(3):637-640. 